\title{
ELLERMAN BOMBS AT HIGH RESOLUTION. I. MORPHOLOGICAL EVIDENCE FOR PHOTOSPHERIC RECONNECTION
}

\author{
Hiroko Watanabe ${ }^{1}$, Gregal Vissers ${ }^{2}$, Reizaburo Kitai $^{1}$, Luc Rouppe van der Voort ${ }^{2}$, And Robert J. Rutten ${ }^{2,3}$ \\ ${ }^{1}$ Kwasan and Hida Observatories, Kyoto University, Yamashina-ku, Kyoto 607-8417, Japan; watanabe@kwasan.kyoto-u.ac.jp \\ ${ }^{2}$ Institute of Theoretical Astrophysics, University of Oslo, P.O. Box 1029 Blindern, N-0315 Oslo, Norway \\ ${ }^{3}$ Sterrekundig Instituut, Utrecht University, Postbus 80000, NL-3508 TA, Utrecht, The Netherlands \\ Received 2011 February 1; accepted 2011 May 2; published 2011 July 6
}

\begin{abstract}
High-resolution imaging-spectroscopy movies of solar active region NOAA 10998 obtained with the Crisp Imaging Spectropolarimeter at the Swedish 1-m Solar Telescope show very bright, rapidly flickering, flame-like features that appear intermittently in the wings of the Balmer $\mathrm{H} \alpha$ line in a region with moat flows and likely some flux emergence. They show up at regular $\mathrm{H} \alpha$ blue-wing bright points that outline the magnetic network, but flare upward with much larger brightness and distinct "jet" morphology seen from aside in the limbward view of these movies. We classify these features as Ellerman bombs and present a morphological study of their appearance at the unprecedented spatial, temporal, and spectral resolution of these observations. The bombs appear along the magnetic network with footpoint extents up to $900 \mathrm{~km}$. They show apparent travel away from the spot along the pre-existing network at speeds of about $1 \mathrm{~km} \mathrm{~s}^{-1}$. The bombs flare repetitively with much rapid variation at timescales of seconds only, in the form of upward jet-shaped brightness features. These reach heights of 600-1200 km and tend to show blueshifts; some show bi-directional Doppler signature and some seem accompanied with an H $\alpha$ surge. They are not seen in the core of $\mathrm{H} \alpha$ due to shielding by overlying chromospheric fibrils. The network where they originate has normal properties. The morphology of these jets strongly supports deep-seated photospheric reconnection of emergent or moat-driven magnetic flux with pre-existing strong vertical network fields as the mechanism underlying the Ellerman bomb phenomenon.
\end{abstract}

Key words: Sun: activity - Sun: atmosphere - Sun: magnetic topology

Online-only material: animations

\section{INTRODUCTION}

Ellerman bombs are prominent, sudden, short-lived brightness enhancements of the outer wings of strong optical lines, in particular the Balmer $\mathrm{H} \alpha$ line at $\lambda=6563 \AA$, that occur in solar active regions. In his discovery paper, Ellerman (1917, p. 298) described them as "a very brilliant and very narrow band extending four or five angstroms on either side of the $[\mathrm{H} \alpha]$ line, but not crossing it," fading after a few minutes. He named them "solar hydrogen bombs"; nowadays, they are named after him. They occur exclusively in emerging flux regions, are very bright, and have a characteristic elongated shape at sufficient angular resolution.

The fairly extensive literature on Ellerman bombs is reviewed in a parallel paper (R. J. Rutten et al. 2011, in preparation, henceforth Paper I) which also addresses the issue that not all bright features in the wings of $\mathrm{H} \alpha$ are necessarily Ellerman bombs (or "moustaches," another name often taken for the same phenomenon). Small photospheric concentrations of strong magnetic field of the type that constitute network and plage can also produce strikingly bright points in the wings of $\mathrm{H} \alpha$, the blue wing in particular (Leenaarts et al. 2006a, 2006b). We refer to Paper I for this issue and for a wider overview of the Ellerman bomb literature. The older literature was summarized well in the introduction of Georgoulis et al. (2002). A recent review of small-scale photospheric magnetic fields is given by de Wijn et al. (2009).

Selected recent Ellerman bomb studies of particular relevance here, in addition to the comprehensive study of Georgoulis et al. (2002), are those by Pariat et al. (2004, 2007), Isobe et al. (2007), Watanabe et al. (2008), Matsumoto et al.
(2008), Hashimoto et al. (2010), Morita et al. (2010), and Guglielmino et al. (2010). These studies provide evidence that the Ellerman bomb phenomenon occurs at sites of and is due to magnetic reconnection, and characteristically has elongated shapes reminiscent of the "chromospheric anemone jets" of Shibata et al. (2007). The present paper strengthens this case on the basis of $\mathrm{H} \alpha$ observations that are far superior in quality to any in the literature, and establishes that Ellerman bombs of the type seen here are rooted in the deep photosphere. In the remainder of this introduction, we show and discuss a few illustrative examples, and then report more comprehensive measurements in the main body of the paper.

Our observations, described in detail in Section 2, were obtained with the Crisp Imaging Spectropolarimeter (CRISP) at the Swedish 1-m Solar Telescope (SST) on La Palma. The target was an active region with a spot and extended plage where flux was still emerging, seen limbward at viewing angle $\mu=0.67$. A 37 minute sequence of high-resolution images sampling 23 wavelengths covering $\mathrm{H} \alpha$ was taken at a temporal cadence of $6.2 \mathrm{~s}$ between consecutive $\mathrm{H} \alpha$ profile scans.

The observations can be inspected as animations in the online edition of the journal. They show a few dozen eruptive features that are very bright in the $\mathrm{H} \alpha$ wings and appear as rapidly varying extended flames or jets, jutting out from photospheric network, pointing away from disk center. These are clearly Ellerman bombs, seen at unprecedented spatial and temporal resolution. The limbward viewing helps to display their upward extent and fast variations as seen from the side. It also diminishes the network bright point contrast so that the bombs stand out more clearly (cf. Paper I). Figures 1-3 discussed below show cutout snapshots that serve as 

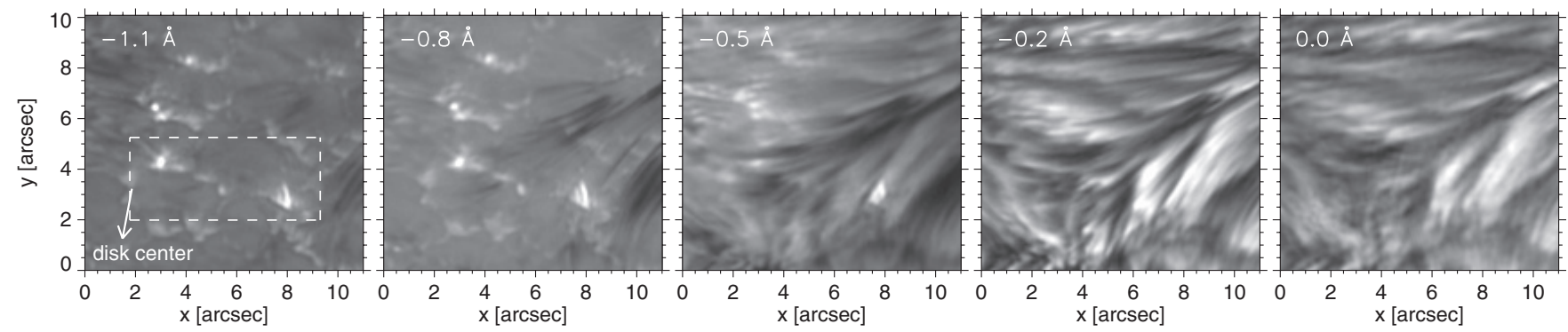

Figure 1. Sample of Ellerman bombs in the small subfield of the CRISP profile scan taken at 08:01 UT outlined in the upper left panel of Figure 4. Clockwise rotation over $120^{\circ}$ from $(X, Y)$ was applied to obtain local $(x, y)$ coordinates with the limbward (vertical) direction nearly upward; the direction to disk center is shown in the first panel. The spot lies to the left; nearby plage lies to the right. The five images sample $\mathrm{H} \alpha$ at $\Delta \lambda=-1.1,-0.8,-0.5,-0.2$, and $0.0 \AA$ from line center. Each panel is gray scaled separately. The dashed white frame in the first panel outlines the yet smaller subfield used in Figure 2 . An animation of $-1.1,-0.5$, and $0.0 \AA$ is available in the online edition of the journal. Full field-of-view animations of -1.1 and $0.0 \AA$ are also available.

(Animations $[\mathrm{A}, \mathrm{B}, \mathrm{C}]$ of this figure is available in the online journal.)
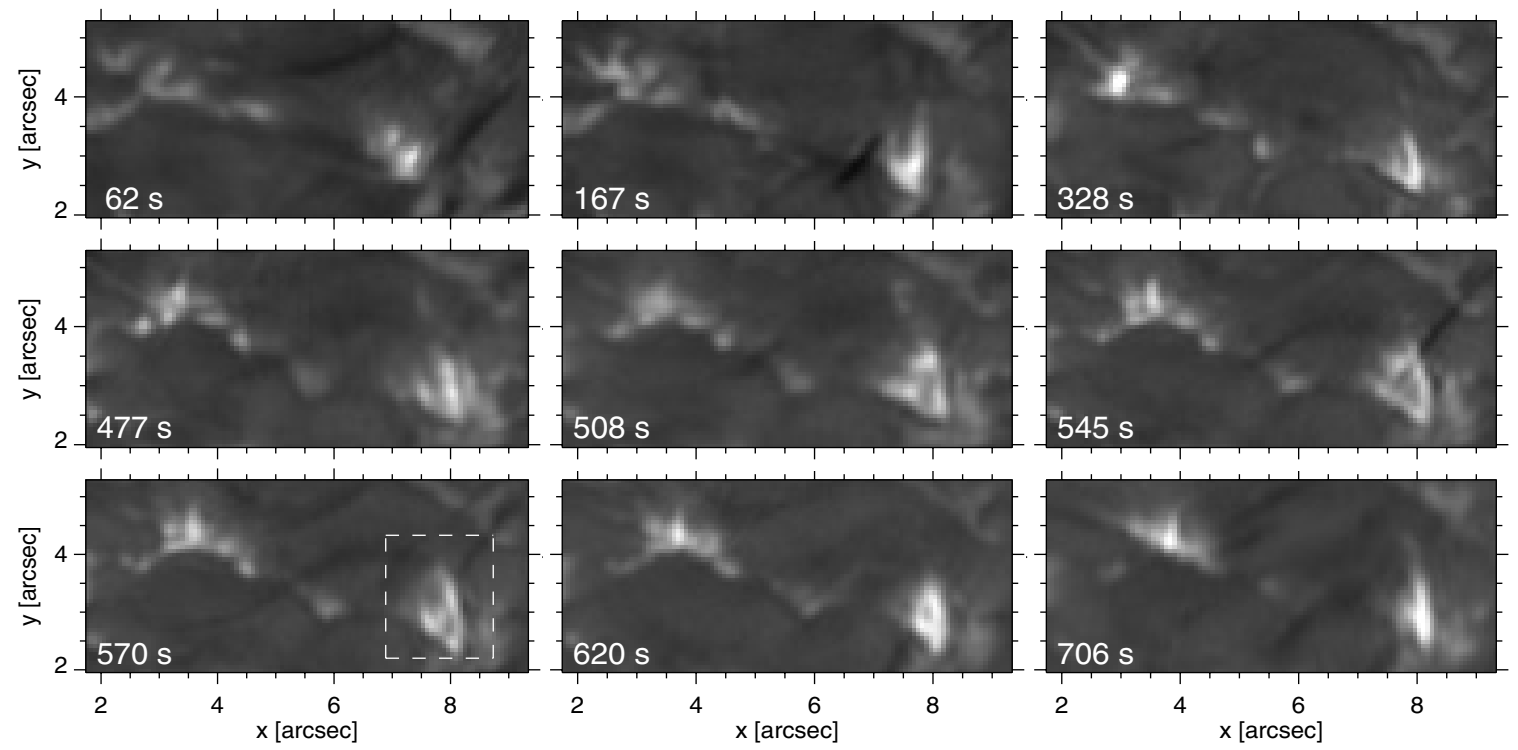

Figure 2. Ellerman bomb evolution in a selection of CRISP image cutouts at $\Delta \lambda=-1.1 \AA$ from $\mathrm{H} \alpha$ line center. The small subfield shown here is outlined in Figure 1 and contains the most prominent Ellerman bomb (EB-1) at $(x, y)=(8,3)$. The elapsed time from 07:55 UT is specified in each panel and increases in unequal steps along rows. The $t=328 \mathrm{~s}$ panel corresponds to the samples in Figures 1, 4, and 5. The dashed white frame specifies the EB-1 cutout shown at $2 \mathrm{~s}$ cadence in Figure 3.
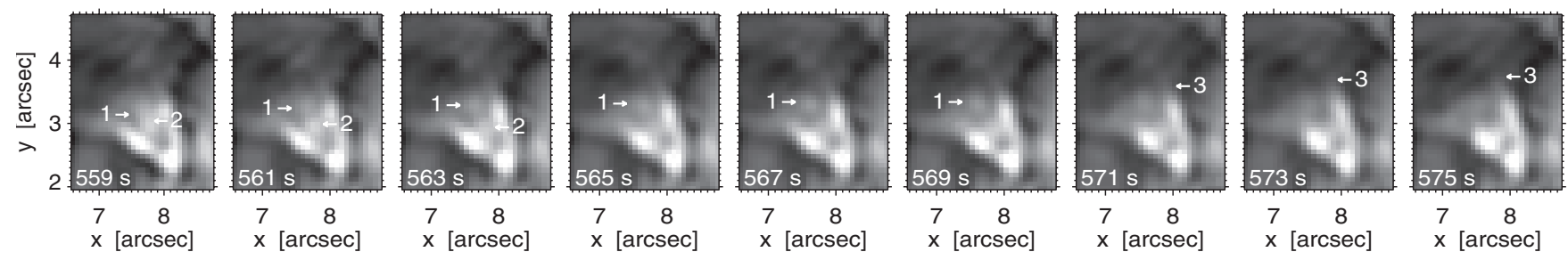

Figure 3. Rapid variations in Ellerman bomb EB-1 in simultaneously taken wide-band images at $2 \mathrm{~s}$ cadence. The subfield contains only EB-1. It is outlined in the seventh panel of Figure 2 which is straddled by the brief sequence shown here. The elapsed time specified in the lower left corner of each panel corresponds to that in Figure 2. Upward motion of brightness features is marked comoving arrow 1, downward motion with arrow 2, and extension of an elongated jet structure with arrow 3. (An animation of this figure is available in the online journal.)

a quick-look emulation of viewing the Ellerman bombs in these animations, but we strongly recommend that the reader inspect the animations.

Ellerman bomb visibility. Figure 1, which samples the deep photosphere, demonstrates, for a small subfield, why Ellerman bombs appear only in the outer wings of $\mathrm{H} \alpha$, apart from rare Doppler-shifted fibrils (Leenaarts et al. 2006b; Paper I). Closer to line center, they are fully shielded from view by the overlying opaque $\mathrm{H} \alpha$ chromosphere made up of dark fibrils. Note that each panel is gray scaled separately for best contrast; in intensity units, the brightest features at line center are much darker than the Ellerman bombs in the wings, so these do not poke through the fibril canopy even though they extend to heights normally considered chromospheric. Comparing the line-wing and line-center animations in the online material confirms that the line-center fibril evolution is unrelated to the Ellerman bomb activity underneath, except the few Ellerman bombs that are accompanied by an $\mathrm{H} \alpha$ surge.

Ellerman bomb evolution. Figure 2 samples the time evolution of the yet smaller subfield specified in Figure 1. The largest Ellerman bomb is designated EB-1 henceforth. It migrates to the right, along the network away from the spot, 
with consecutive flaring-up of adjacent threads that reach a large extent with apparently near-vertical orientation. At some instances, for example at $t=328 \mathrm{~s}$, the bright threads have thicker bright feet suggesting small-scale "inverted-Y" (or "Tour d'Eiffel") morphology. The Ellerman bomb site at $(x, y)=(3.5,4.5)$ shows a similar sequence of smaller thread-like brightenings migrating to the right along the network.

Figure 3 samples even faster variations of EB-1 in frames taken simultaneously with the CRISP sequence but at much higher cadence, with another camera registering $\mathrm{H} \alpha$ with a $4.9 \AA$ wide passband. In order to enhance the contrast of interesting features in the still images, an unsharp masking method similar to the procedure described by Hashimoto et al. (2010), but using a Gaussian filter width of 0.5 arcsec, was employed. The figure demonstrates that small-scale brightness features within EB-1 change and move on timescales of seconds. The apparent speeds are measured in Section 4 and are supersonic.

Ellerman bomb properties. Viewing the animations establishes a number of key properties of the Ellerman bombs in these data as follows.

1. These Ellerman bombs are all rooted in intergranular lanes in the deep photosphere.

2. They all have upward protrusions that we generically call "jets" here, often appearing in the form of nearly parallel or consecutive slender bright threads.

3. They all point away from disk center in the limbward field of view, and so appear to be more or less vertically oriented. None seems to sense the more horizontal orientation of the overlying chromospheric fibrils that are observed at the center of $\mathrm{H} \alpha$.

4. The Ellerman bombs migrate, while flaring repetitively, along the pre-existing network, always away from the spot.

5. There are very fast variations in their fine structure during the repetitive flaring.

6. Some of the brighter threads display small-scale inverted-Y morphology.

7. Some Ellerman bombs are accompanied by dark $\mathrm{H} \alpha$ surges.

Together, these properties define the Ellerman bombs in this data set as photospheric events rooted in the network that produce eruptive jets reaching upward to considerable height, with fast temporal variations that suggest successive interaction of the newly arriving vertical field with the existing network field, most likely in the form of magnetic reconnection. Photospheric reconnection has been suggested theoretically (cf. Shibata et al. 1982; Yokoyama \& Shibata 1995; Litvinenko 1999) but has not been established observationally. The present observations provide the clearest evidence to date.

In the rest of the paper, we detail the observations (Section 2) and the methods used for the selection and measurements of Ellerman bombs and other bright points (Section 3). In Section 4, we quantify the observed Ellerman bomb footpoint motions, extensions and retractions, their Doppler signatures, and their fast variations. We also compare Ellerman bomb properties with those of non-eruptive network bright points and display one of the two cases with a surge. We discuss the Ellerman bomb phenomenon in Section 5, including a brief comparison with previous studies, and conclude the paper in Section 6.

\section{OBSERVATIONS AND DATA REDUCTION}

Instrumentation. The data for this study were obtained with the CRISP (Scharmer et al. 2008) at the SST (Scharmer et al. 2003a) on La Palma. CRISP is a dual Fabry-Pérot interferometer allowing wavelength tuning within $50 \mathrm{~ms}$. Its combination with the unprecedented angular resolution and image sequence quality attained by the SST's superb optics and site combined with sophisticated wavefront restoration yields data of outstanding spatial, temporal, and spectral resolution (e.g., Rouppe van der Voort et al. 2009; Ortiz et al. 2010). The SST real-time tip-tilt correction and adaptive-optics wavefront correction are described by Scharmer et al. (2003b). The postdetection numerical image restoration is described by van Noort et al. (2005).

Observations. The setup of CRISP starts with an optical chopper synchronizing the exposures by multiple cameras. A filterwheel contains appropriate prefilters; in this case, one was used that selects $\mathrm{H} \alpha$ with a passband of FWHM $=4.9 \AA$. A few percent of the light is branched off to a camera taking images in this wide band. These serve usually only for "multiobject" seeing monitoring in the numerical post-processing, but in this study they were also analyzed because of their fast cadence. The bulk of the light passes through two liquid crystals and a high-resolution and a low-resolution Fabry-Pérot etalon, and is then divided by a polarizing beam splitter between two cameras. The three CCD cameras are high-speed, low-noise Sarnov CAM1M100 ones with $1 \mathrm{~K} \times 1 \mathrm{~K}$ chips. They ran synchronously at 35 frames per second with an exposure time of $17 \mathrm{~ms}$. The image scale was 0.071 arcsec pixel ${ }^{-1}$, well sampling the SST's Rayleigh diffraction limit of $0.17 \operatorname{arcsec}$ at $\mathrm{H} \alpha$. The field of view covered $67 \times 67 \operatorname{arcsec}^{2}$. The CRISP transmission profile has $\mathrm{FWHM}=6.6 \mathrm{pm}$ at $\mathrm{H} \alpha$.

An $\mathrm{H} \alpha$ sequence was acquired between 07:55 and 08:33 UT on 2008 June 11 during good to excellent seeing conditions. The target was AR 10998, containing a leading sunspot with a rudimentary penumbra and following plages. The sunspot appeared on 2008 June 8, kept growing until June 11, and had fully disintegrated by June 15 . In these June 11 data, the region was located at viewing angle $\mu=0.67$ toward the east limb. The field of view was centered on $(X, Y) \approx(-684,-152)$ with $(X, Y)$ the standard solar coordinates in arcsec from apparent disk center with $Y$ positive along the solar meridian toward the north pole, $X$ positive westward.

The $\mathrm{H} \alpha$ profile was sequentially sampled at 23 wavelengths within the line, ranging between $\pm 1.1 \AA$ with respect to line center with $0.1 \AA$ spacing. At each tuning position, a "multiframe" burst of eight exposures was taken. The cadence between consecutive line scans was $6.2 \mathrm{~s}$.

Another observing program was run before and after the $\mathrm{H} \alpha$ observations, targeting the same area but sampling the magnetically sensitive Fe I 6302 Å lines. It ran during 07:15-07:32 UT and 09:26-10:26 UT. The first run had CRISP sampling both the $6301.5 \AA$ and $6302.5 \AA$ lines as well as a continuum wavelength, at a scan cadence of $41 \mathrm{~s}$. The later run sampled only the $6302.5 \AA$ line plus a continuum wavelength at a scan cadence of $16 \mathrm{~s}$. Simple Stokes- $V / I$ magnetograms were constructed from the flat-fielded images at $\Delta \lambda=-48 \mathrm{~m} \AA$ from line center, selected for high Stokes- $V$ signal and large contrast, for inspection of the magnetic context prior to and after the $\mathrm{H} \alpha$ observations. One such magnetogram is shown in Figure 4.

Reduction. The post-processing to achieve further image restoration including precise co-alignment was done with the Multi-Object Multi-Frame Blind Deconvolution (MOMFBD) algorithm of van Noort et al. (2005). It includes tessellation of all images (i.e., at each tuning position within a line scan) into $64 \times 64$ pixel $^{2}$ subfields for individual restoration. The wide- 

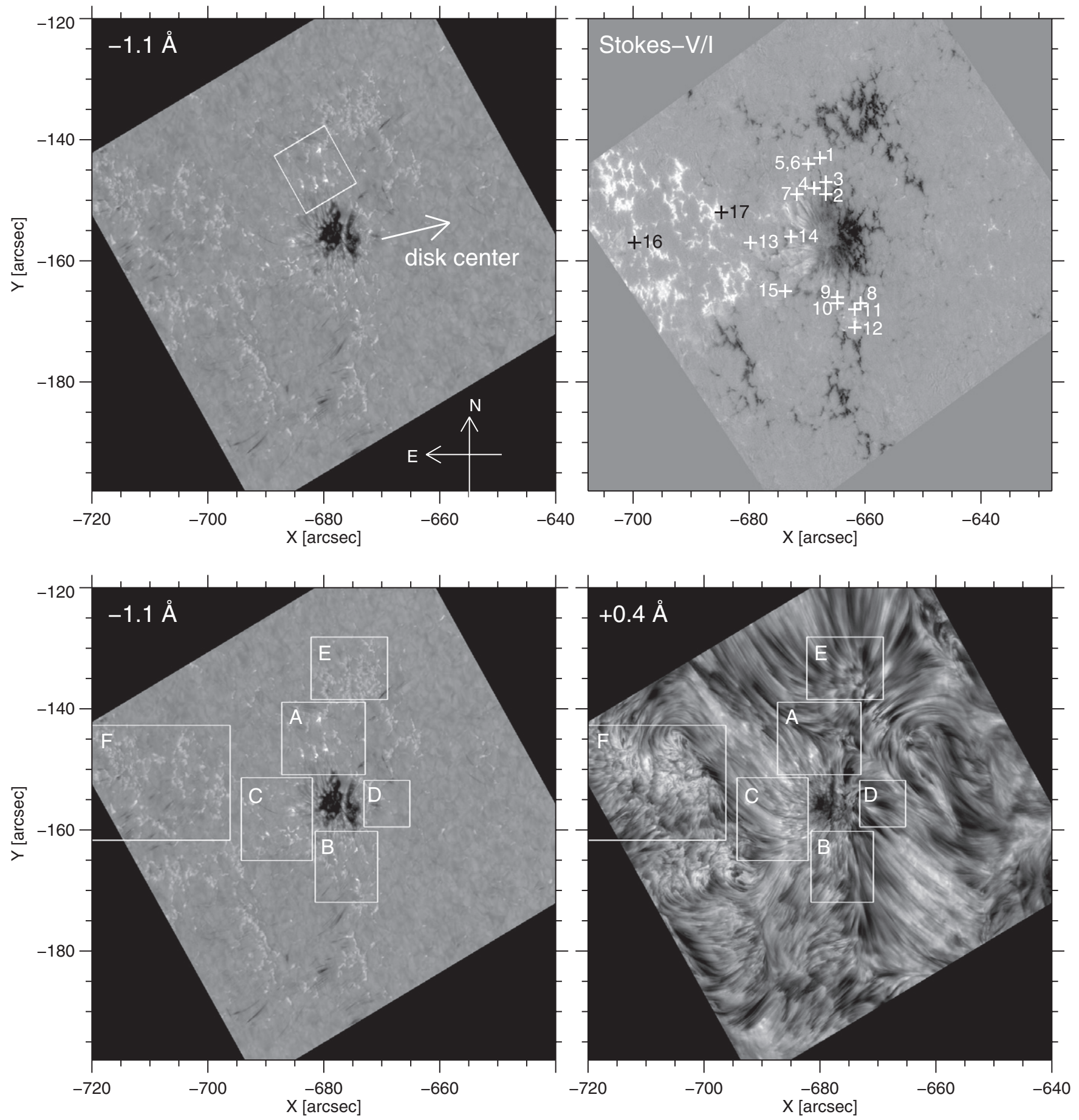

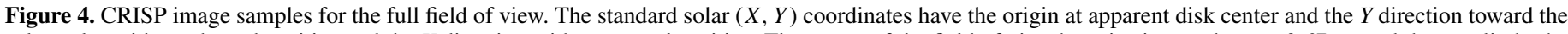
solar poles with northward positive and the $X$ direction with westward positive. The center of the field of view has viewing angle $\mu=0.67$ toward the east limb; the direction toward disk center is indicated in the first panel. The field of view includes AR 10998. Upper left panel: $\mathrm{H} \alpha-1.1 \AA ̊$ blue-wing image taken at 08:01 UT. The white frame specifies the subfield that is shown in Figure 1 with clockwise rotation over $120^{\circ}$. The brightest feature in this subfield is Ellerman bomb EB-1, shown in detail in Figures 2 and 3. Upper right panel: a CRISP Fe I $6302 \AA$ Stokes- $V / I$ magnetogram taken two hours later, with corresponding shift in $X$ due to solar rotation. The numbered plus signs define the locations of the 17 Ellerman bombs measured in Section 3. Lower left panel: the same H $\alpha-1.1 \AA$ blue-wing image overlaid with frames specifying the subregions selected for bright-point measurements in Section 3. Lower right panel: simultaneous H $\alpha+0.4 \AA$ image overlaid with the same subregion frames.

band data served both as multi-object representation and as an anchor for alignment of the narrow-band bursts per sequence. In this case the wide-band frames were also processed separately from the CRISP data to obtain a simultaneous sequence at a cadence of only $1 \mathrm{~s}$, which serves below to analyze fast temporal evolution within Ellerman bomb EB-1 (Figure 3). More detail on
MOMFBD reconstruction strategies for such data sets is given in van Noort \& Rouppe van der Voort (2008).

Subsequently, the restored images were subjected to prefilter correction removing the transmission profile of the wide-band filter, to derotation which removes the time-varying image rotation that results from the alt-azimuth configuration of the 
SST, and to destretching which removes remaining rubber-sheet distortions (small-scale translations and warping) by seeing. These are determined following Shine et al. (1994) through cross-correlation of running means over a few minutes of small subfields of the wide-band sequence and they are then applied to the narrow-band images.

Figure 4 shows two full-field samples, for the outer blue wing at $\Delta \lambda=-1.1 \AA$ from line center in the left-hand column and for the inner red wing at $\Delta \lambda=+0.4 \AA$ from line center in the lower right panel, respectively. Both are from the CRISP scan at 08:01 UT, a moment of very good seeing that yielded the largest image contrast of the 37 minute sequence. The upper right panel contains a corresponding magnetogram from the Fe I $6302 \AA$ data taken two hours later. The various figure annotations are described below.

\section{FEATURE SELECTION AND MEASUREMENT}

\subsection{Ellerman Bombs}

Occurrence in the field of view. Inspection of the various $\mathrm{H} \alpha$-wing animations shows the occurrence of many Ellerman bombs near the spot, in particular to the east where there was an extended plage of opposite polarity (upper panels of Figure 4). Note that the penumbra shows black-and-white bipolar difference between the east and west sides of the spot, but this results from the combination of slanted viewing and near-horizontal penumbral field configuration. The plage fields are primarily vertical; their black/white signature in the magnetogram in Figure 4 indeed specifies opposite polarity. Most Ellerman bombs appear fairly close to the spot.

Spectral variations. Figures 1 and 5 illustrate the spectral behavior of $\mathrm{H} \alpha$ over the target area. Figure 1 samples the small subfield outlined in Figure 4 at five wavelengths in the blue $\mathrm{H} \alpha$ wing. The wing images $(\Delta \lambda=-1.1 \AA,-0.8 \AA)$ show a grayish background with somewhat brighter network. The photospheric contribution to this part of $\mathrm{H} \alpha$ originates in the deep photosphere, but in the blue wing the granular contrast is flattened as explained by Leenaarts et al. (2006b). The network contrast diminishes toward the limb, as explained in Paper I. The Ellerman bombs appear very bright. Figure 5, which samples $\mathrm{H} \alpha$ profiles for different pixel categories, shows that in Ellerman bombs the $\mathrm{H} \alpha$ wings reach nearly twice the intensity they have in quiet-Sun areas and extend well beyond our $\Delta \lambda= \pm 1.1 \AA$ sampling range - in accordance with Ellerman's (1917) remark that they extend $4-5 \AA$ on either side.

At line center, all Ellerman bombs are hidden by overlapping chromospheric fibrils (or "mottles"; we use the term fibril generically for long slender filamentary structures). The second panel of Figure 1 shows the onset of this obscuration. At $\Delta \lambda=-0.8 \AA$, only a few dark fibrils appear, as was also the case in the observations of Leenaarts et al. (2006b) at the same wavelength. These isolated dark fibrils are mostly chromospheric absorption features with large blueshift, probably the "Rapid Blue Excursions" that Rouppe van der Voort et al. (2009) identified as the on-disk counterpart of so-called spicules-II seen at the solar limb. Closer to line center, the chromospheric fibrils gain opacity and together form an opaque blanket obscuring any photospheric contribution underneath, including the upright jets of the Ellerman bombs. At $\Delta \lambda=-0.5 \AA$, all brightest areas in Figure 1 still correspond to the underlying Ellerman bombs, although not $1: 1$ in shape, but at $\Delta \lambda=-0.2 \AA$ this is no longer true. Even the tallest Ellerman bomb in Figure 1, EB-1, is obscured at line center.
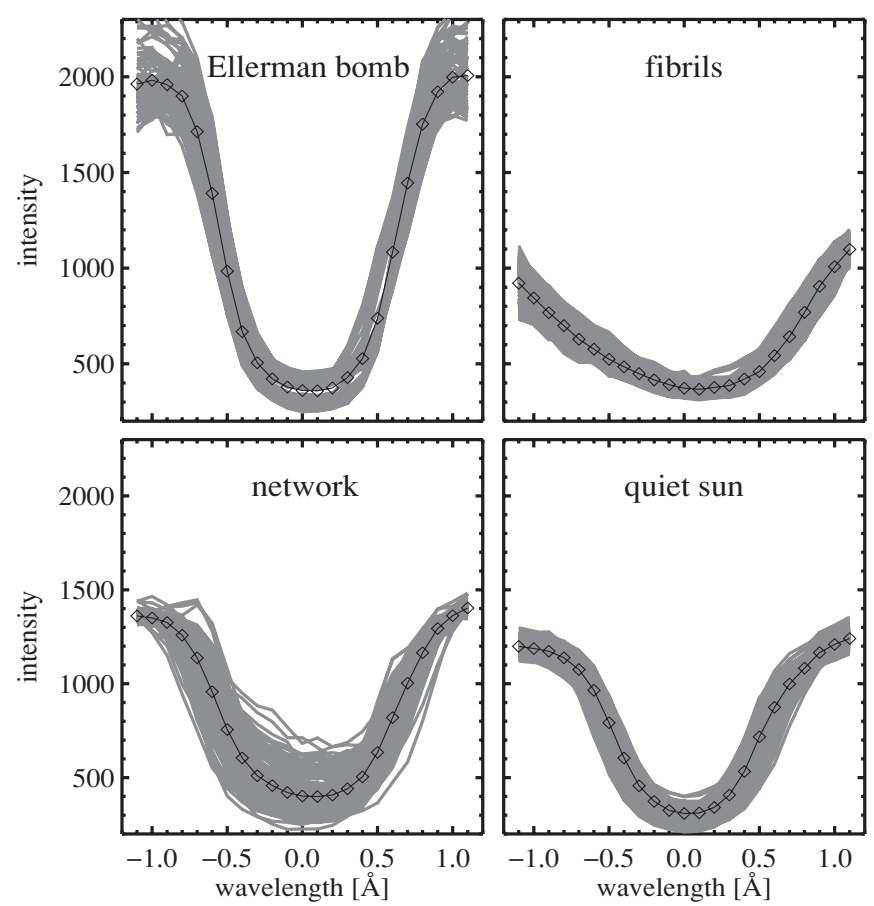

Figure 5. Characteristic $\mathrm{H} \alpha$ profiles collected from the CRISP scan taken at 08:01 UT. Each panel contains a number of per-pixel profiles for a specific pixel category. The solid curve is their mean, with the CRISP sampling wavelengths marked. The intensity scale (in arbitrary data units) is the same for the different categories. The samples were selected by first obtaining the average wing brightness $\left\langle I_{\text {wing }}\right\rangle$ per pixel by summing the blue- and red-wing values at $\Delta \lambda=-1.1,-1.0,-0.9,+0.9,+1.0,+1.1 \AA$. Clockwise from top left: Ellerman bombs, all 84 pixels with $\left\langle I_{\text {wing }}\right\rangle>1800$; fibrils, 314 pixels with $\left\langle I_{\text {wing }}\right\rangle<950$ in the eastern part of the field of view; quiet Sun, 250 pixels covering a small area in the region around $(X, Y)=(-665,-170)$ in Figure 4; network without Ellerman bombs, all 169 pixels with $1349<\left\langle I_{\text {wing }}\right\rangle<1350$. Note that at the $\Delta \lambda= \pm 1.1 \AA$ sampling extremes the mean profile reaches only about $70 \%$ of the continuum intensity for a quiet-Sun average.

Correspondingly, the $\mathrm{H} \alpha$ cores in Figure 5 are as dark for the Ellerman bomb locations as they are for dark fibrils that are selected as such on the basis of dark outer wings. Obviously, the fibril obscuration determines this part of the Ellerman bomb profiles. Since Ellerman bombs always occur in emerging flux regions that necessarily possess rich fibril structure in the overlying $\mathrm{H} \alpha$ chromosphere, obscuration-free Ellerman bomb observation at $\mathrm{H} \alpha$ line center may inherently be impossible.

Selection. The animations suggest that the Ellerman bombs are relatively long-lived brightness entities, appearing repetitively while their feet migrate along the network. We decided to use the outer $\mathrm{H} \alpha$ wings as a measurement diagnostic by defining their sum and difference as $I_{\text {sum }} \equiv\left(I_{r}+I_{b}\right) / 2$ and $I_{\text {diff }} \equiv\left(I_{r}-I_{b}\right) /\left(I_{r}+I_{b}\right)$, with $I_{b}$ the spectrally averaged blue-wing intensity at $\Delta \lambda=-1.1,-1.0$, and $-0.9 \AA$ from line center and, likewise, $I_{r}$ the averaged red-wing intensity at $\Delta \lambda=+1.1,+1.0$, and $+0.9 \AA$ from line center. The outerwing averaging reduces noise from seeing variations. The wing summing and differencing removes, to first order, the effect of Dopplershift on $I_{\text {sum }}$ and the effect of intensity change on $I_{\text {diff }}$. We treat the wing difference as a Doppler signal below. This is a better choice for Ellerman bombs than measuring the profile-minimum shift or core bisector shift, which are set by the overlying fibrils. For emission features such as Ellerman bombs, positive $I_{\text {diff }}$ implies redshift. Since the Ellerman bombs appear to extend upright, the measured redshifts imply downflows at the $\mu=0.67$ viewing angle. 

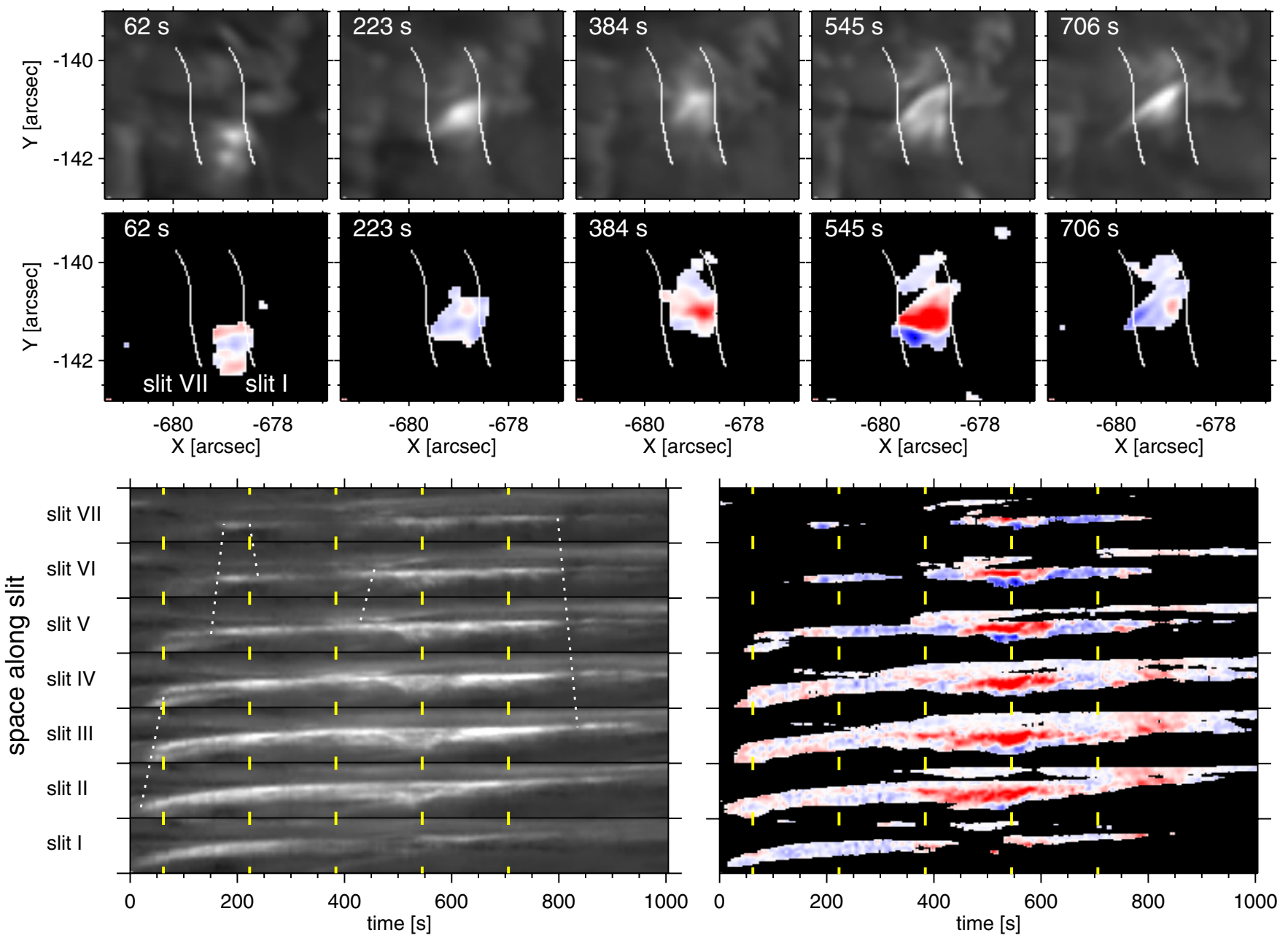

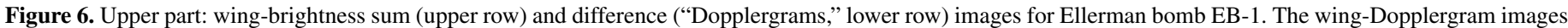

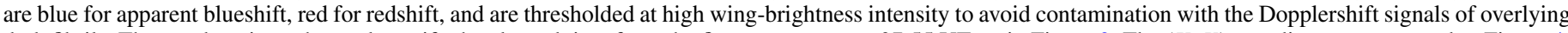

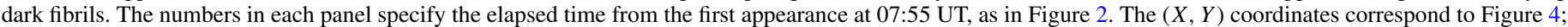

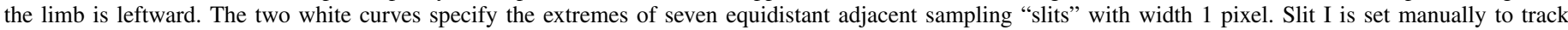

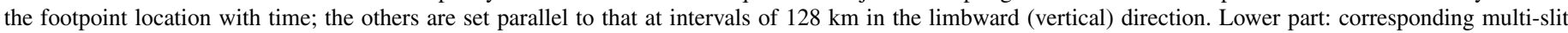

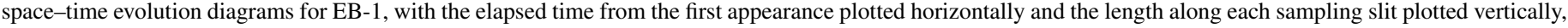

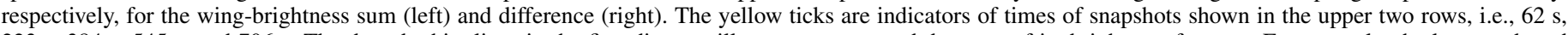

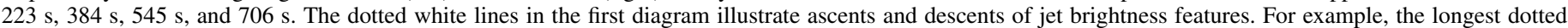

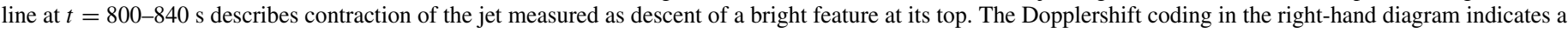
bi-directional jet with upward motion (blueshift) of its upper part and downward motion (redshift) of its lower part during $t=500-750 \mathrm{~s}$.

(An animation of this figure is available in the online journal.)

We constructed $I_{\text {sum }}$ wing-brightness and $I_{\text {diff }}$ wingDopplergram images for the whole data sequence. We used the $I_{\text {sum }}$ sequence to identify and select Ellerman bombs manually, using excess wing brightness, presence of a jet-like protrusion, and visibility above $240 \mathrm{~s}$ duration as criteria. Thus we selected 17 Ellerman bombs whose positions are indicated in the second panel of Figure 4. The first 15 lie in the periphery of the spot or the moat around it (recognizable from the outward moat flow across it in the animations). EB-16 and EB-17 lie at the edge of a plage. Overall, these Ellerman bombs seem to be located preferentially at the network and moat boundaries. We found no Ellerman bombs at the more quiescent west side of the spot.

Space-time slit diagrams. We have constructed what we call "multi-slit space-time diagrams" for all 17 Ellerman bombs to facilitate quantitative measurement of their apparent footpoint proper motion, the extension and retraction speeds of their jetlike protrusions, and the sign and magnitude of their Doppler signals. These measurements are non-trivial due to the motion that the Ellerman bombs display along the network while flaring up in a succession of jet-like or thread-like protrusions. Figure 6 shows such a diagram for EB-1.

The measurement procedure is as follows. First, the approximate jet direction (N, NE, E,...) is determined by eye. Second, a baseline spatial "slit" (called slit I) of 1 pixel width is defined manually such that it contains the bottom edge, or footpoint, of the protrusion and tracks its subsequent locations; this track is generally not straight. Third, six more slits II-VII are positioned by spacing them parallel to slit I, sharing the same bends, at every $128 \mathrm{~km}$ in the approximate jet direction. Slit VII is then the furthest from the footpoint, at $768 \mathrm{~km}$ from slit I. Together, these slits represent samplings of the jet along its apparent height that track its footpoint path. They enable the construction of space-time plots for the wing brightness $I_{\text {sum }}$ and wing Dopplershift $I_{\text {diff. }}$. In the latter, we found it necessary to mask off all areas that have wing brightness below 1400 


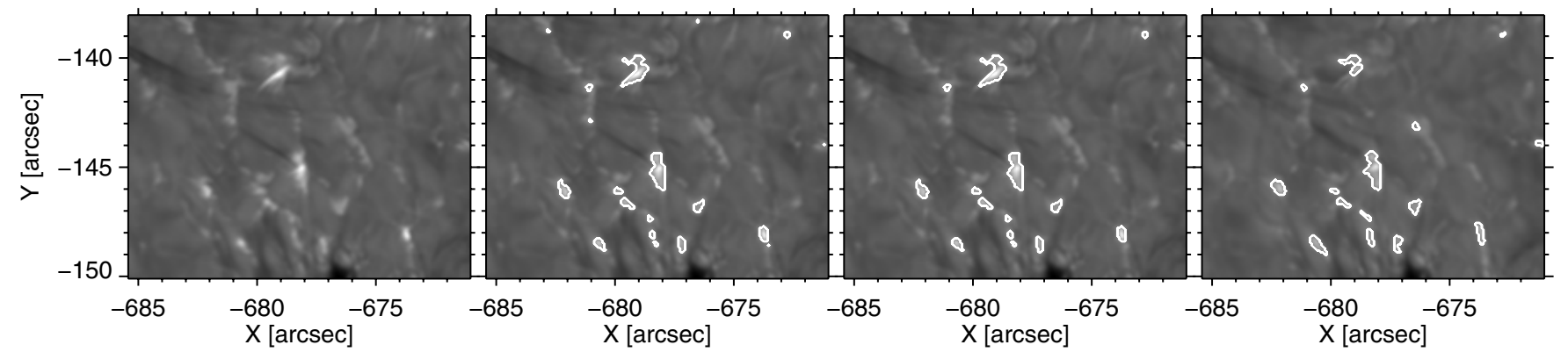

Figure 7. Automatic detection of network bright points using the wing-brightness images. First panel: Region A at 08:08 UT. The $(X, Y)$ coordinates correspond to Figure 4. Second panel: areas over $3 \sigma$ brighter than the average values are outlined with white contours. Third panel: result after removal of small bright points. Fourth panel: a similar map one minute later, illustrating good continuity.

Table 1

Ellerman Bomb Properties

\begin{tabular}{|c|c|c|c|c|c|c|c|c|}
\hline EB & $\begin{array}{c}\text { Jet } \\
\text { Direction }\end{array}$ & $\begin{array}{l}\text { Lifetime } \\
\text { (s) }\end{array}$ & $\begin{array}{l}\text { Footpoint } \\
\text { Extent }^{\mathrm{a}} \\
(\mathrm{km})\end{array}$ & $\begin{array}{c}\text { Jet } \\
\text { Extent }^{\mathrm{a}, \mathrm{b}} \\
(\mathrm{km})\end{array}$ & $\begin{array}{c}\text { Footpoint } \\
\text { Speed } \\
\left(\mathrm{km} \mathrm{s}^{-1}\right)\end{array}$ & $\begin{array}{c}\text { Extension } \\
\text { Speed }^{\mathrm{b}} \\
\left(\mathrm{km} \mathrm{s}^{-1}\right)\end{array}$ & $\begin{array}{l}\text { Retraction } \\
\text { Speed }^{\mathrm{b}} \\
\left(\mathrm{km} \mathrm{s}^{-1}\right)\end{array}$ & $\begin{array}{c}\text { Blue/Red } \\
\text { Signal at } \\
\text { J:jet, F:footpoint }\end{array}$ \\
\hline 1 & SE & 1004 & 820 & 1180 & 1.23 & $5.5-9.8$ & 5.9 & J:blue, F:red \\
\hline 2 & $\mathrm{E}$ & 508 & 360 & 770 & 1.15 & 6.2 & 9.8 & no \\
\hline 3 & $\mathrm{E}$ & 1302 & 390 & 690 & 1.13 & 6.2 & 5.6 & J:blue \\
\hline 4 & $\mathrm{E}$ & 713 & 460 & 770 & 1.00 & 7.6 & $\ldots$ & $\mathrm{J}$ :blue \\
\hline 5 & $\mathrm{E}$ & 260 & 280 & 1230 & no & 5.7 & $4.6-6.4$ & J:blue, F:red \\
\hline 6 & $\mathrm{E}$ & 266 & 280 & 1120 & no & 11.0 & 8.7 & J:blue, F:red \\
\hline 7 & $\mathrm{E}$ & 359 & 490 & 870 & 1.60 & $6.4-8.3$ & 6.9 & J:red, F:blue \\
\hline 8 & $\mathrm{E}$ & 682 & 310 & 750 & 1.31 & $3.8-4.2$ & 10.4 & $\mathrm{~F}$ : blue, then red \\
\hline 9 & $\mathrm{E}$ & 322 & 620 & 540 & 1.11 & 23.7 & $\ldots$ & $\mathrm{J}:$ blue \\
\hline 10 & $\mathrm{E}$ & 651 & 510 & 850 & 1.44 & $7.2-8.3$ & 11.9 & J:blue, F:blue \\
\hline 11 & $\mathrm{E}$ & 428 & 690 & 670 & 0.85 & 18.5 & $\ldots$ & J:red \\
\hline 12 & $\mathrm{NE}$ & 459 & 330 & 1050 & 0.29 & $\ldots$ & $8.5-15.1$ & F,J: red, then blue \\
\hline 13 & $\mathrm{~N}$ & 279 & 900 & 720 & no & $6.4-11.9$ & 6.4 & J:blue, F:red \\
\hline 14 & $\mathrm{E}$ & 868 & 440 & 950 & 0.27 & $2.8-3.5$ & 9.2 & J:red, F:blue \\
\hline 15 & $\mathrm{E}$ & 589 & 410 & 800 & no & 2.9 & $\ldots$ & no \\
\hline 16 & SE & 614 & 410 & 930 & no & $6.4-11.9$ & 11.9 & $\mathrm{~J}$ : red, then blue \\
\hline 17 & $\mathrm{E}$ & 322 & 310 & 640 & no & 6.2 & $\ldots$ & J:red, F:blue \\
\hline Average & $\mathrm{E}$ & 566 & 471 & 855 & 0.67 & 8.0 & 8.7 & $\ldots{ }^{\mathrm{c}}$ \\
\hline
\end{tabular}

Notes.

a The apparent footpoint measured on best-seeing images.

${ }^{b}$ If a jet is vertical, which they are roughly, the actual lengths and velocities are a factor of 1.5 larger.

c Too much spread to give a meaningful average.

(in the arbitrary data units also used in Figure 5) because otherwise, overlying chromospheric fibrils dominate the Doppler signature with their independent Dopplershifts. In addition, for these dark features the Dopplergram definition switches sign, causing extra confusion, as does their varying viewing angle with respect to the line of sight. By masking the dark fibrils off, the space-time Doppler signature is much better constrained to display Ellerman bomb properties only. Finally, using these multi-slit space-time diagrams we derived the proper-motion speed of all Ellerman bomb feet, the extension and retraction speeds of all jets, and the spatial occurrence distributions along all Ellerman bombs of their Doppler signatures. The results are given in Section 4 and Table 1.

\subsection{Network Bright Points}

In order to compare the characteristics between Ellerman bombs and normal network bright points (henceforth NBP), we also analyzed the latter in our data sequence. Like the Ellerman bombs, the NBPs are not distributed uniformly within the field of view but occur preferentially to the east of the spot (Figure 4). In order to compare NBP properties between different types of region, six subregions named A-F were chosen that are identified in the lower panels of Figure 4. Regions A, B, and $\mathrm{C}$ sample the moat flow around the spot on its north, east, and south sides. Region C contains the well-developed part of the penumbra, whereas Region D samples the penumbra-free west side of the spot. Region E samples the same-polarity plage to the north of the spot, whereas Region F samples the oppositepolarity plage to the east of the spot.

We applied a brightness threshold algorithm to identify NBPs for statistical analysis using the wing-brightness images. A similar algorithm was developed for the detection of umbral dots in Watanabe et al. (2009). Note that Ellerman bombs are also NBPs when categorized per brightness threshold, but the Ellerman bombs defined and selected above possess eruptive jets as well. The procedure consists of four steps illustrated in Figure 7.

1. Identify NBPs in each image. NBPs are defined as areas whose wing-sum brightness $\left(I_{\text {sum }}\right)$ is over $3 \sigma$ brighter than the average of the pertinent subregion, with $\sigma$ the standard deviation. For plage regions a threshold of $5 \sigma$ is used instead. The latter selects only a few NBPs in plage that usually lie at the plage periphery. A lower threshold would select much of the plage as NBP, without individual 
properties or measurable morphology. We then defined the position of each NBP as the peak location within the thresholded area.

2. Remove too small NBPs. Too small NBPs covering less than 5 pixels ( 0.18 arcsec in radius) are removed. Note that the diffraction limit is 0.17 arcsec.

3. Track NBPs. An NBP at a subsequent time step is considered to mark the continued manifestation of a prior NBP if they have spatial overlap. We accepted the cases in which such continuation skipped one or two time steps. When there is no overlap within three successive time steps, the NBP is considered to have disappeared.

4. Remove the merging/splitting events. The procedures described above include some merging or splitting events. We remove these events by selecting the longest-duration event and separate it from merging/splitting pieces. Thus, one of two merging or splitting pieces is kept for tracking while the other ends or starts at this time step.

For example in Region A, a total of 183 NBPs were detected in this manner. Seven of these were also selected as Ellerman bombs in Section 3.1.

\section{RESULTS}

\subsection{Ellerman Bomb Properties}

Our measurements of the 17 selected Ellerman bombs are given in Table 1. Their footpoints travel lengths along the network and their jet extents were measured on best-seeing images. The resulting jet extents and the jet extension and retraction speeds are apparent lengths and velocities. If the jets are vertical, which they roughly seem to be, the real values are larger by a factor $1 / \mu=1.5$. The mean apparent jet extent is close to $1 \mathrm{Mm}$, so the jets stick out well above the photosphere. The mean lifetime is about 10 minutes.

Footpoint motion. The wing-brightness distribution across slit II in the space-time diagrams such as the one for EB-1 in Figure 6 was used for the measurement of footpoint proper motion because this slit usually contains the brightest part of the bright point at the foot of an Ellerman bomb. When there is proper motion, the space-time diagram shows an inclined bright lane, as is the case in Figure 6. The inclination was then measured and converted into apparent speed. The direction of motion is always radially away from the spot. The average speed of footpoint motion of Ellerman bombs is $0.67 \mathrm{~km} \mathrm{~s}^{-1}$.

Extension and retraction speed. We identified apparent jet extensions or retractions using the wing-brightness panels of the multi-slit space-time diagrams together with the corresponding movie. Some examples are marked in Figure 6 with white dotted lines. Their slope gives the extension/retraction speed by dividing the pertinent slit separation in units of their $128 \mathrm{~km}$ spacing by the travel time. The typical value is $5-10 \mathrm{~km} \mathrm{~s}^{-1}$, without systematic difference between the extension and retraction speeds. Of course, the actual gas speed depends on the projection; for vertical jets, the measured apparent speeds should be multiplied by $1 / \mu=1.5$.

Dopplershift. We found interesting Dopplershift signatures in the wing-Dopplergram space-time charts in 15 cases out of 17 Ellerman bombs. EB-1 in Figure 6 starts with subtle blueshift and later displays simultaneous redshift at the footpoint NBP and blueshift near the jet top during $500 \mathrm{~s}-750 \mathrm{~s}$. Three more cases show such combination of footpoint redshift with jet blueshift. The pattern suggests the presence of a bi-directional jet, with downflow at its bottom and upflow at its top. The

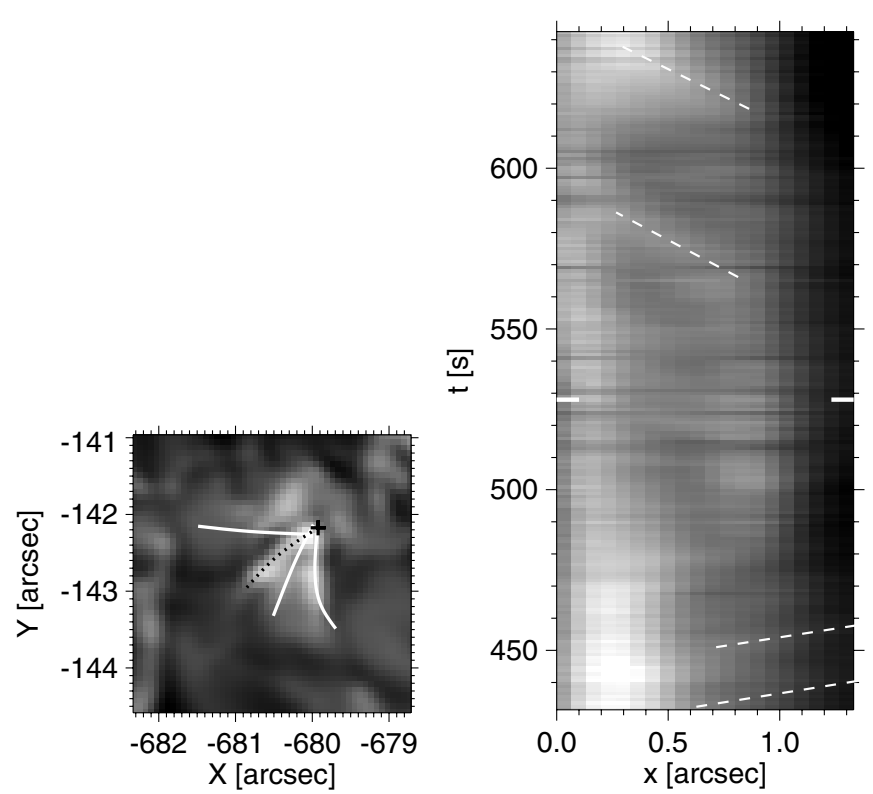

Figure 8. Left: measurement tracks through Ellerman bomb EB-1 in the wideband data at 08:04 UT. The $(X, Y)$ coordinates correspond to Figure 4. Right: time-space diagram along the black dotted track in the left-hand image, with the elapsed time from the first appearance plotted vertically. The 0 arcsec location is indicated in the right panel with a black plus sign. The slanted dotted lines specify apparent-speed estimations, while the white tick marks at $t \approx 530 \mathrm{~s}$ indicate the time step at which sample data are shown in the right panel.

blueshifts tend to last longer than the redshifts. In three cases we found the opposite pattern: downflow at the top and upflow at the bottom. Sometimes an Ellerman bomb alternates between the two patterns during its lifetime. In the two cases where the jet is clearly accompanied by a surge (EB-8 and EB12, discussed below), the latter's Dopplershift obscures the underlying Ellerman bomb motion. In two cases (EB-2 and EB15), we could not find Ellerman bomb associated Dopplershifts, probably due to interweaving upflow and downflow components or seeing perturbation.

Fast variations. Figure 3 and the wide-band movie in the online material demonstrate that, at the $1 \mathrm{~s}$ cadence of the wideband image sequence, very fast variations are observed. As residual high-frequency seeing effects might contaminate the sample of real fast variations, a low-pass filter with a cutoff of $50 \mathrm{~km} \mathrm{~s}^{-1}$ was applied to the wide-band data. In addition, only those variations that were visible in more than one subsequent frame were considered for the analysis described below, while residual seeing effects are typically only visible for one frame and furthermore random in nature.

A more detailed analysis of the fast variations was performed for EB-1. First, the cutout subfield frames were cross-correlated to remove EB-1's proper motion. Time-space diagrams were then extracted along paths that track in time either the jet top or single bright features inside the Ellerman bomb. The lefthand panel of Figure 8 shows the selected paths overlaid on a good quality EB-1 image. The right-hand panel shows part of the time-space diagram along the dashed path. The timeslices enable measurement of the apparent proper-motion speed for particular features that move along these paths, as well as the jet extension and retraction speed. Four such measurements have been overlaid as dashed lines in the right panel in Figure 8 to illustrate the procedure. The upper two concern downdraft features, both with an apparent speed around $18 \mathrm{~km} \mathrm{~s}^{-1}$, 

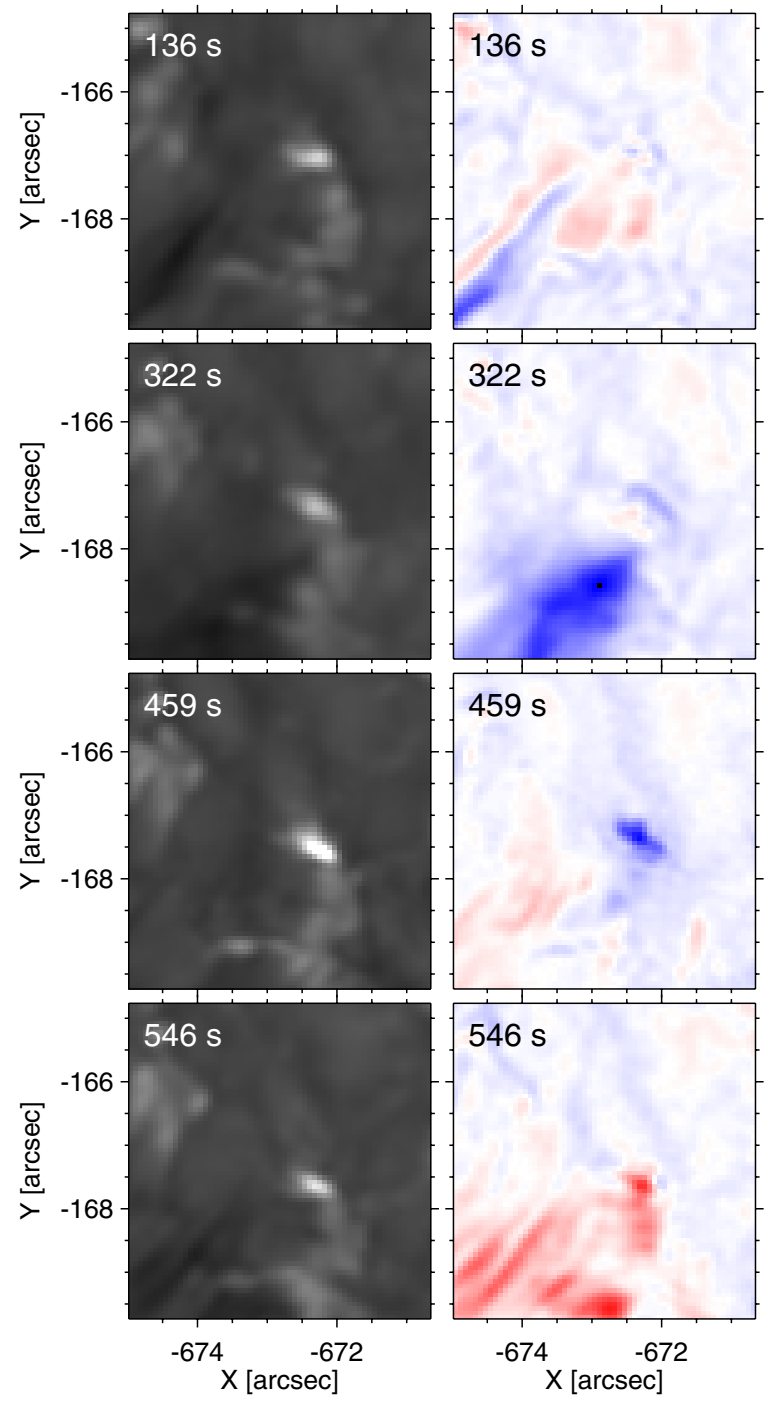

Figure 9. Temporal evolution of Ellerman bomb EB-8 in wing-brightness (left) and wing-Dopplergram images (right). The numbers in each panel specify the elapsed time from its first appearance. The $(X, Y)$ coordinates correspond to Figure 4 . The limb is to the left. This Ellerman bomb seems to initiate a dark extension to its southeast (lower left direction), with alternating Dopplershift signature (where blue color implies downflow for the absorption feature.)

whereas the lower two are updrafts, with apparent speeds close to $59 \mathrm{~km} \mathrm{~s}^{-1}$.

In this manner 11 displacement speed measurements were obtained for EB-1. The typical timescale for jet-like features to extend/retract is $25-40 \mathrm{~s}$, but isolated brightness features can exist on timescales of 2-7 s. Six out of 11 cases display updrafts and the rest display downdrafts. The apparent updraft speeds range between 11 and $60 \mathrm{~km} \mathrm{~s}^{-1}$ with the majority at the higher values, while the apparent downdraft speed range between 7 and $18 \mathrm{~km} \mathrm{~s}^{-1}$.

Surges. Figure 9 shows the case of EB- 8 which displays special temporal evolution. First, the Ellerman bomb appears and seems to initiate surge activation on its southeast side. This surge appears repetitively during the observations, with alternating negative and positive $I_{\text {diff }}$. Note that, since the surge is observed as an absorption feature in $\mathrm{H} \alpha$, the Dopplershift sign is reversed with respect to the Ellerman bomb which is observed in emission, so that for the surge, positive wing difference $I_{\text {diff }}$ implies upflow along the line of sight, i.e.,
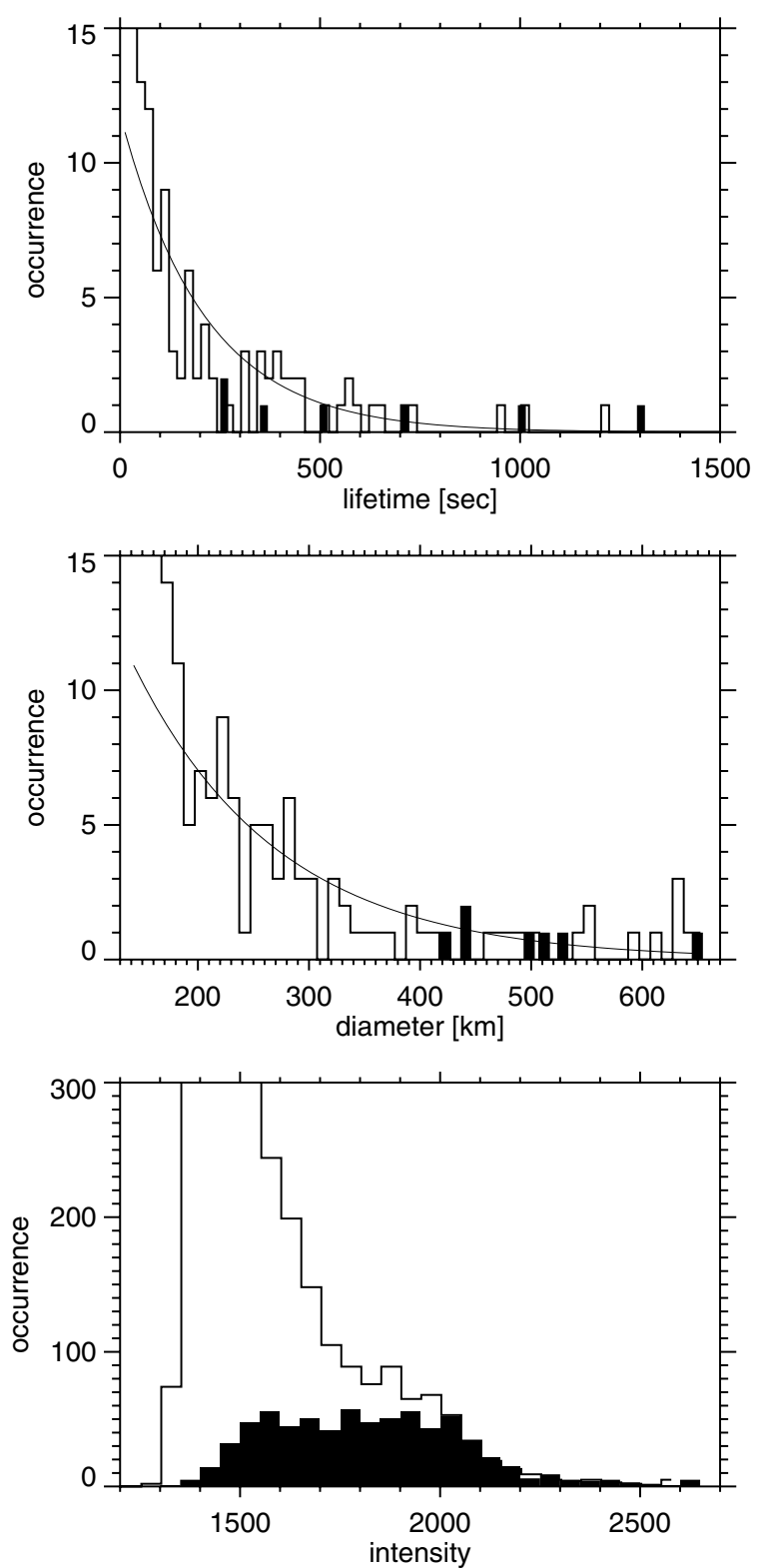

Figure 10. Histograms of bright-point lifetime, diameter, and brightness in Region A. The seven Ellerman bombs found in this region are entered as black bins. The curves in the upper two panels are fits with exponential decay functions $y=$ const. $\times \exp (-x / C)$.

blueshift although it is color-coded red in Figure 9. The surge exhibits strong positive $I_{\text {diff }}$ corresponding to an upflow of $\approx 40 \mathrm{~km} \mathrm{~s}^{-1}$ (measured from the $\mathrm{H} \alpha$ line-center Doppler shift) at around $322 \mathrm{~s}$ after the appearance of EB-8. About 1 minute after the surge disappears, negative $I_{\text {diff }}$ (downflow) appeared at the position of the Ellerman bomb, and 1 minute after that positive $I_{\text {diff }}$ (upflow) replaced the negative $I_{\text {diff. }}$. Simultaneously, the surge location was also dominated by redshift.

EB-12 is similarly adjacent to an $\mathrm{H} \alpha$ surge on its south side, which also shows alternating positive and negative $I_{\text {diff }}$.

\subsection{Ellerman Bombs versus Normal NBPs}

Figure 10 shows histograms of the lifetime, diameter, and wing brightness of NBPs in Region A. The seven NBPs that are also Ellerman bombs are entered as black bins. The diameter is calculated by assuming circular shape, converting its area into the corresponding circle diameter, and taking the average over 
Table 2

NBP Properties

\begin{tabular}{|c|c|c|c|c|c|}
\hline Region & $\begin{array}{c}\text { Total Number } \\
\text { of NBPs Detected }\end{array}$ & $\begin{array}{l}\text { Characteristic } \\
\text { Lifetime (s) }\end{array}$ & $\begin{array}{l}\text { Characteristic } \\
\text { Diameter }(\mathrm{km})\end{array}$ & $\begin{array}{l}\text { Average Proper } \\
\text { Speed }\left(\mathrm{km} \mathrm{s}^{-1}\right)\end{array}$ & $\begin{array}{l}\text { Occurrence Rate } \\
\left(\operatorname{arcsec}^{-2} \min ^{-1}\right)\end{array}$ \\
\hline A & 183 & 222 & 273 & 0.88 & 0.028 \\
\hline B & 141 & 232 & 214 & 0.75 & 0.030 \\
\hline $\mathrm{C}$ & 206 & 240 & 255 & 0.83 & 0.033 \\
\hline $\mathrm{D}$ & 89 & 173 & 225 & 0.59 & 0.039 \\
\hline $\mathrm{E}$ & 41 & 111 & 205 & $\ldots{ }^{a}$ & 0.002 \\
\hline $\mathrm{F}$ & 6 & $\ldots{ }^{a}$ & $\ldots{ }^{a}$ & $\ldots{ }^{a}$ & 0.001 \\
\hline
\end{tabular}

Note. ${ }^{\text {a }}$ Too few samples to calculate.

the NBP lifetime. The histogram for brightness contains values from every time step, making the number of samples large. The detection thresholds for the lifetime and diameter measurement were $6.2 \mathrm{~s}$ and $135 \mathrm{~km}$, respectively. The brightness threshold is set by the NBP detection algorithm (Section 3.2).

As for other automated detection methods (Sobotka et al. 1997; Watanabe et al. 2009), these histograms show exponential decrease. We fit the first two histograms with functions $y \propto$ $\exp (-x / C)$, with $C$ the characteristic value. In the case of Region A, a characteristic lifetime of $222 \mathrm{~s}$ and characteristic diameter of $273 \mathrm{~km}$ were obtained. The only striking difference between Ellerman bombs and normal NBPs is in the brightness panel, Ellerman bombs being extraordinary bright in the $\mathrm{H} \alpha$ wings - commensurate with their definition-but with overlap during their startup and decay phases, which makes the presence of a jet a necessary discriminator between Ellerman bombs and the brightest NBPs. We conclude that, apart from their brightness, Ellerman bombs are just like non-eruptive NBPs.

Table 2 shows the average proper-motion speed and occurrence rate of the NBPs in the six subregions. The average propermotion speed was determined from NBPs with a lifetime longer than $60 \mathrm{~s}$ by dividing the distance between the disappearance and appearance locations by its lifetime, and taking the average of those for each region. The occurrence rate is defined by the average NBP occurrence frequency within one square arcsec area per minute.

Table 2 suggests a subdivision into three types of regions. First, Regions A, B, and C can be characterized as moat flow regions in which NBPs are relatively long-lived and large, moving with the same speed as that of the moat flow. Second, the occurrence rate in Region D, covering the outside of a penumbrafree area, is highest, but the NBPs do not move much and their lifetimes are relatively short. The appearance of NBPs in this region follows the intergranular lane morphology. Third, Region $\mathrm{E}$ and $\mathrm{F}$ sampling plages contain only a few individual NBPs that fit our detection algorithm. These lie mostly at the plage peripheries. The other bright features within the plages are too crowded for our detection scheme so that we cannot measure their characteristic lifetimes and diameters, but they tend to be smaller and more static.

\section{DISCUSSION}

Ellerman bomb occurrence, type, mechanism. Most Ellerman bombs in our data appear on the east side of the spot that has a well-developed penumbra. They seem to take part in the moat flow that emanates from the penumbra in their fast proper motion along the network (see Vargas Domínguez et al. 2007, 2008 for evidence that moat flows require penumbrae). In particular, the repetitive brightening of jet threads and the jet direction (away from disk center in the limbward field of view) suggests that new flux with near-vertical orientation arrives at the network as driven by the moat flow, and reconnects with the pre-existing, also largely vertical, network fields. The brightenings start low and extend rapidly upward in jet-like flames, sometimes evolving into bi-directional jets. The flows at the location of Ellerman bombs alternately change sign and the jet lengths extend and retract repetitively. This behavior indicates reconnection that takes place again and again by encountering preexisting magnetic field. The highly supersonic apparent feature speeds measured from the wide-band data suggest phase speed rather than material motion, reminiscent of the shear motion described by van Ballegooijen et al. (1998).

The recent literature discusses multiple types of Ellerman bombs. In particular, Pariat et al. (2004, 2007) and Watanabe et al. (2008) found evidence for undulating "serpentine" field topologies with Ellerman bombs formed at the dip of field lines (called "bald patches" in the French literature), where the fields are nearly antiparallel. The fact that Ellerman bombs are reported to occur only in emerging flux regions speaks for an antiparallel reconnection scenario. The repetitivity of the bright-thread appearance while the Ellerman bomb migrates along the network may indeed result from sea-serpent field topology, but located in the photosphere rather than extending to the chromosphere.

Since we have no simultaneous magnetogram data at similar angular resolution, we can only speculate about the magnetic topology of our Ellerman bombs. There may be oppositepolarity field emergence with direct or with separatrix/separator reconnection (cf. Parnell \& Galsgaard 2004), or there may be component reconnection between unipolar fields that shear along each other in differential moat flows. In our case, we did observe considerable field topology changes at the east side of the spot during the two magnetogram sequences taken before and after the $\mathrm{H} \alpha$ sequence, but this gives only an indication that further field emergence may well have taken place during the $\mathrm{H} \alpha$ sequence. We think it most likely that the Ellerman bombs in our data require a field topology that produces nearvertical reconnection deep in the photosphere, and we suspect that the moat flow is a key operator. The strong differential moat flow combined with the presence of a nearby bipolar plage might suffice as condition to produce our type of Ellerman bomb.

Recently, Guglielmino et al. (2010) reported an SST plus Hinode observation of a flux emergence region exhibiting an event much like our Ellerman bombs and accompanied by an $\mathrm{H} \alpha$ surge. They attribute it, with the help of simulation examples, to reconnection due to interaction between emerging flux and the pre-existing chromospheric and coronal field. In contrast, in our Ellerman bombs the reconnective interaction seems to 
be fully defined within the photosphere, although 2 of our 17 Ellerman bombs do have an accompanying surge.

Photospheric formation. All Ellerman bombs in our $\mathrm{H} \alpha$ images originate from intergranular lanes, i.e., from regular granulation in the deep photosphere that is sampled by the $\mathrm{H} \alpha$ wings apart from occasional Doppler-shifted absorption features (Leenaarts et al. 2006b; Rouppe van der Voort et al. 2009; Paper I). The reversed granulation at heights $h=100-200 \mathrm{~km}$ is seen in $\mathrm{H} \alpha$ only closer to line center in extremely quiet fibril-free regions. All our Ellerman bombs lie below the chromospheric fibril canopy. $\mathrm{H} \alpha$ is commonly thought to be chromospheric; for example, Georgoulis et al. (2002) described their Ellerman bombs as low-chromosphere features on the basis of their detection in filtergrams at $\Delta \lambda=-0.8 \AA$ from $\mathrm{H} \alpha$ line center which suggests that these were actually as photospheric as ours. Therefore, our observations strengthen the conclusion of Georgoulis et al. (2002) that Ellerman bombs are probably a signature of low-altitude reconnection.

Similarity to chromospheric anemone jets. All our Ellerman bombs have extended jets; in fact, the jet presence became a selection criterion after our initial animation inspections. Shibata et al. (2007) reported the existence of so-called chromospheric anemone jets seen in Ca II $\mathrm{H}$ with the Hinode Solar Optical Telescope. They have lengths of 2000-5000 km and apparent extension speeds of $10-20 \mathrm{~km} \mathrm{~s}^{-1}$. They are found preferentially in mixed-polarity regions (Morita et al. 2010). In particular, in a sunspot moat, Morita et al. (2010) found nine such chromospheric anemone jets with lifetimes ranging between 30 and $320 \mathrm{~s}$, in good agreement with our Ellerman bomb durations. Some of our Ellerman bombs also show the inverted "Y" shape characteristic of anemone jets, and also the apparent jet extension speeds are comparable. Morita et al. (2010) attributed the jet extension speed to the Alfvén velocity in the low chromosphere; we note that the Alfvén velocity in the photosphere (at field strength $1000 \mathrm{G}$ and density $10^{17} \mathrm{~cm}^{-3}$ ) is comparable. The major difference is that the Ellerman bomb jets tend to reach smaller heights. Nevertheless, we suggest that our type of Ellerman bombs and chromospheric anemone jets represent the same phenomenon, with the difference in $\mathrm{H} \alpha$ and $\mathrm{Ca}$ II $\mathrm{H}$ extent perhaps due to the difference in fibril visibility in the two diagnostics.

Ellerman bomb heating. Our data suggest strongly that our Ellerman bombs are caused by magnetic reconnection of strong near-vertical network fields with also near-vertical field that is probably moat-flow driven into the network. Undoubtedly there is sizable heating. There are attempts to model the bright $\mathrm{H} \alpha$ wings of Ellerman bombs by adding appropriate temperature humps to standard models in one-dimensional modeling or inversion (Socas-Navarro et al. 2006; Berlicki et al. 2010), but these are likely to underestimate the upward extent of the heating by trying to also reproduce the dark $\mathrm{H} \alpha$ core which in reality is caused by opaque chromospheric fibrils that are not taken into account. We suspect that exceedingly large thermal line width, as proposed by Matsumoto et al. (2008) and particular to the Balmer lines due to the small atomic mass of hydrogen, may be an important aspect. $\mathrm{H} \alpha$ line synthesis in numerical magnetohydrodynamic simulations including encounter or close-encounter reconnection is obviously desirable.

\section{CONCLUSION}

The imaging spectroscopy presented in this paper is far superior to any $\mathrm{H} \alpha$ observation of Ellerman bombs in the literature. It yielded unprecedented spatial and temporal resolution that we exploited to describe the structure and evolution of the 17 Ellerman bombs seen in the $\mathrm{H} \alpha$ wings. They are all rooted deeply in the magnetic network on the side of a sunspot with strong moat flow, and they all have upright jets that reach considerable height, although these jets remain shielded by overlying chromospheric fibrils at $\mathrm{H} \alpha$ line center.

Although multiple types of Ellerman bombs and corresponding field configurations are reported in the literature, the ones reported here all seem to be photospheric events that are most likely explained by successive reconnection of strong vertical network fields with incoming, also vertical, field that is driven into the network by the moat flow.

The $\mathrm{H} \alpha$ observations presented in this paper have one obvious shortcoming: there was no simultaneous polarimetry performed that would provide corresponding magnetograms at similar angular resolution. Such data are required to study the topology and evolution of the magnetic fields at and around Ellerman bomb sites to ascertain what happens magnetically, in particular to establish the presence and action of field emergence and cancellation and to chart the field polarity. Note that such mapping should be photospheric. Chromospheric magnetometry is currently a grail of solar spectropolarimetry, but for Ellerman bombs such as the ones discussed here it would diagnose the field of overlying fibrils, not the bombs or their triggering, and be irrelevant unless the Ellerman bomb sends off a measurable surge.

In 2010, the Oslo group obtained new SST/CRISP sequences that combine $\mathrm{H} \alpha$ and $\mathrm{Ca} 8542$ line scans with $\mathrm{Fe}_{\mathrm{I}} 6302$ spectropolarimetry of a sunspot in an emerging flux region yet closer to the limb. These data are well suited for further study, presently underway, of the Ellerman bomb phenomenon.

We thank our colleagues at the Kwasan and Hida Observatories and at Kyoto University and Oslo University for fruitful discussions. This research was supported by a Grant-in-Aid for JSPS Fellows, a Grant-in-Aid for the Global COE Program "The Next Generation of Physics, Spun from Universality and Emergence" of the Ministry of Education, Culture, Sports, Science and Technology (MEXT) of Japan, and by the Research Council of Norway through grants 177336/V30 and 191814/V30. The Swedish 1-m Solar Telescope is operated on the island of $\mathrm{La}$ Palma by the Institute for Solar Physics of the Royal Swedish Academy of Sciences in the Spanish Observatorio del Roque de los Muchachos of the Instituto de Astrofísica de Canarias. R.J.R. visited Japan as a guest of NAOJ at Mitaka and acknowledges hospitality at Kyoto University and Hida Observatory.

\section{REFERENCES}

Berlicki, A., Heinzel, P., \& Avrett, E. H. 2010, Mem. Soc. Astron. Ital., 81, 646 de Wijn, A. G., Stenflo, J. O., Solanki, S. K., \& Tsuneta, S. 2009, Space Sci. Rev., 144,275

Ellerman, F. 1917, ApJ, 46, 298

Georgoulis, M. K., Rust, D. M., Bernasconi, P. N., \& Schmieder, B. 2002, ApJ, 575,506

Guglielmino, S. L., Bellot Rubio, L. R., Zuccarello, F., Aulanier, G., Vargas Domínguez, S., \& Kamio, S. 2010, ApJ, 724, 1083

Hashimoto, Y., et al. 2010, PASJ, 62, 879

Isobe, H., Tripathi, D., \& Archontis, V. 2007, ApJ, 657, L53

Leenaarts, J., Rutten, R. J., Carlsson, M., \& Uitenbroek, H. 2006a, A\&A, 452, L15

Leenaarts, J., Rutten, R. J., Sütterlin, P., Carlsson, M., \& Uitenbroek, H. 2006b, A\&A, 449, 1209

Litvinenko, Y. E. 1999, ApJ, 515, 435

Matsumoto, T., Kitai, R., Shibata, K., Otsuji, K., Naruse, T., Shiota, D., \& Takasaki, H. 2008, PASJ, 60, 95 
Morita, S., Shibata, K., Ueno, S., Ichimoto, K., Kitai, R., \& Otsuji, K. 2010 PASJ, 62, 901

Ortiz, A., Bellot Rubio, L. R., \& Rouppe van der Voort, L. 2010, ApJ, 713, 1282

Pariat, E., Aulanier, G., Schmieder, B., Georgoulis, M. K., Rust, D. M., \& Bernasconi, P. N. 2004, ApJ, 614, 1099

Pariat, E., Schmieder, B., Berlicki, A., Deng, Y., Mein, N., López Ariste, A., \& Wang, S. 2007, A\&A, 473, 279

Parnell, C. E., \& Galsgaard, K. 2004, A\&A, 428, 595

Rouppe van der Voort, L., Leenaarts, J., de Pontieu, B., Carlsson, M., \& Vissers, G. 2009, ApJ, 705, 272

Scharmer, G. B., Bjelksjo, K., Korhonen, T. K., Lindberg, B., \& Petterson, B. 2003a, Proc. SPIE, 4853, 341

Scharmer, G. B., Dettori, P. M., Lofdahl, M. G., \& Shand, M. 2003b, Proc. SPIE, 4853, 370

Scharmer, G. B., et al. 2008, ApJ, 689, L69

Shibata, K., Nishikawa, T., Kitai, R., \& Suematsu, Y. 1982, Sol. Phys., 77, 121

Shibata, K., et al. 2007, Science, 318, 1591
Shine, R. A., Title, A. M., Tarbell, T. D., Smith, K., Frank, Z. A., \& Scharmer, G. 1994, ApJ, 430, 413

Sobotka, M., Brandt, P. N., \& Simon, G. W. 1997, A\&A, 328, 682

Socas-Navarro, H., Martínez Pillet, V., Elmore, D., Pietarila, A., Lites, B. W., \& Manso Sainz, R. 2006, Sol. Phys., 235, 75

van Ballegooijen, A. A., Nisenson, P., Noyes, R. W., Löfdahl, M. G., Stein, R. F., Nordlund, Å., \& Krishnakumar, V. 1998, ApJ, 509, 435

van Noort, M. J., \& Rouppe van der Voort, L. H. M. 2008, A\&A, 489, 429

van Noort, M., Rouppe van der Voort, L., \& Löfdahl, M. G. 2005, Sol. Phys., 228, 191

Vargas Domínguez, S., Bonet, J. A., Martínez Pillet, V., Katsukawa, Y., Kitakoshi, Y., \& Rouppe van der Voort, L. 2007, ApJ, 660, L165

Vargas Domínguez, S., Rouppe van der Voort, L., Bonet, J. A., Martínez Pillet, V., Van Noort, M., \& Katsukawa, Y. 2008, ApJ, 679, 900

Watanabe, H., Kitai, R., \& Ichimoto, K. 2009, ApJ, 702, 1048

Watanabe, H., et al. 2008, ApJ, 684, 736

Yokoyama, T., \& Shibata, K. 1995, Nature, 375, 42 